\title{
Degradation of Titanium Electrodes in the Alternating Polarity Electrolysis
}

\author{
Pavel S. Shlepakov ${ }^{l}$, Ilia V. Uvarov ${ }^{l, *}$, Victor V. Naumov ${ }^{l}$, Leonid A. Mazaletskiy ${ }^{1,2}$, \\ Vitaly B. Svetovoy ${ }^{3,4}$ \\ ${ }^{1}$ Laboratory of Micro- and Nanosystem Technology, Valiev Institute of Physics and Technology of \\ Russian Academy of Sciences, Yaroslavl Branch, Universitetskaya 21, 150007 Yaroslavl, Russia \\ ${ }^{2}$ P.G. Demidov Yaroslavl State University, Sovetskaya 14, 150003 Yaroslavl, Russia \\ ${ }^{3}$ Zernike Institute for Advanced Materials, University of Groningen - Nijenborgh 4, 9747 AG \\ Groningen, The Netherlands \\ ${ }^{4}$ A.N. Frumkin Institute of Physical Chemistry and Electrochemistry RAS, Leninsky pr. 31, 199071 \\ Moscow, Russia \\ *E-mail: i.v.uvarov@bk.ru
}

doi: $10.20964 / 2019.06 .62$

Received: 7 March 2019 / Accepted: 9 April 2019 / Published: 10 May 2019

\begin{abstract}
Electrolysis of water performed by microsecond voltage pulses of alternating polarity has been used to generate nanobubbles in microscopic systems. These nanobubbles exhibit interesting and useful effects, but their production requires a high current density of $>10 \mathrm{~A} / \mathrm{cm}^{2}$. Deposited platinum or gold electrodes cannot withstand these conditions for a long time. Titanium showed the best durability, although it also undergoes degradation. The mechanism of degradation differs from that in usual DC electrolysis and was not previously explored. In this paper, the wear of thin film titanium electrodes fabricated on a silicon substrate by surface micromachining is investigated. The electrodes are tested in the alternating polarity process of various frequencies and durations. They are oxidized during operation, but the spatial distribution and chemical composition of the oxide differ from those observed in normal electrolysis. The strongest oxidation occurs at the edges of the electrodes, while the central part is less involved. At a high frequency of voltage pulses $(400 \mathrm{kHz})$ the electrodes are oxidized much less than at low frequency $(50-100 \mathrm{kHz})$. The oxide grows due to misbalance between periodic oxidation and reduction processes. Internal mechanical stress generated due to oxidation causes degradation of the electrodes.
\end{abstract}

Keywords: Water electrolysis; Alternating polarity; Electrodes; Oxidation; Degradation; 
(C) 2019 The Authors. Published by ESG (www.electrochemsci.org). This article is an open access article distributed under the terms and conditions of the Creative Commons Attribution license (http://creativecommons.org/licenses/by/4.0/). 